\title{
Automated serviceability prediction of NSM strengthened structure using a fuzzy logic expert system
}

\author{
Kh Mahfuz ud Darain ${ }^{\mathrm{a}, \mathrm{b}}$, Mohd Zamin Jumaat ${ }^{\mathrm{a}, *}$, Md. Altab Hossain ${ }^{\mathrm{c}}$, Md. Akter Hosen ${ }^{\mathrm{a}}$,
} M. Obaydullah ${ }^{\text {a }}$, Md. Nazmul Huda ${ }^{\text {a }}$, I. Hossain ${ }^{\text {c }}$

${ }^{a}$ Department of Civil Engineering Faculty of Engineering, University of Malaya, 50603 Kuala Lumpur, Malaysia

${ }^{\mathrm{b}}$ Architecture Discipline, Science, Engineering and Technology School, Khulna University, 9208 Khulna, Bangladesh

'Department of Mechanical Engineering, Faculty of Engineering, University of Malaya, 50603 Kuala Lumpur, Malaysia

A R T I C L E I N F O

Article history:

Available online 9 August 2014

\section{Keywords:}

Steel

CFRP

Deflection

Crack width

Prediction model

Error analysis

\begin{abstract}
A B S T R A C T
This paper presents a simplified model using a fuzzy logic approach for predicting the serviceability of reinforced concrete (RC) beams strengthened with near surface mounted (NSM) reinforcement. Existing analytical models lack proper formulations for the prediction of deflection and crack width in NSM strengthened beams. These existing models are based on the externally bonded reinforcement (EBR) technique with fiber reinforced polymer (FRP) laminates, which presents certain limitations for application in predicting the behavior of NSM strengthened beams. In this study seven NSM strengthened RC beams were statically tested under four point bending load. The test variables were strengthening material (steel or CFRP) and bond length $(1600,1800$ or $1900 \mathrm{~mm})$. For fuzzification, load and bonded length were used as input parameters and the output parameters were deflection and crack width for steel bar and CFRP bar. Experimentally NSM steel strengthened beams showed better performance in terms of crack width and stiffness, although NSM FRP strengthened beams exhibited enhanced strength increment. For all parameters, the relative error of the predicted values was found to be within the acceptable limit (5\%) and the goodness of fit of the predicted values was found to be close to 1.0. Hence, the developed prediction system can be said to have performed satisfactorily.
\end{abstract}

2014 Elsevier Ltd. All rights reserved.

\section{Introduction}

Structural strengthening has become an important area of research as many existing structures underperform and require rehabilitation to meet current standards and service conditions. The near surface mounted (NSM) reinforcement technique has become a popular strengthening method due to its superior ability to increase flexural and shear strength, and to delay or avoid premature debonding failure in comparison to the externally bonded reinforcement (EBR) technique. In the NSM technique, fiber reinforced polymer (FRP) strips or steel rods are inserted into pre-sawn grooves in the concrete cover and bonded therein with epoxy adhesive.

Serviceability refers to the satisfactory performance of a structure under normal service load conditions based on the occupancy type. Deflection and cracking behavior are the most importan parameters when considering the serviceability of a structure. The serviceability of a structure is significantly influenced by

* Corresponding author at: Department of Civil Engineering, University of Malaya, 50603 Kuala Lumpur, Malaysia. Tel.: +60193129194.

E-mail address: zamin@um.edu.my (M.Z. Jumaat).

http://dx.doi.org/10.1016/j.eswa.2014.07.058

0957-4174/@ 2014 Elsevier Ltd. All rights reserved. structural strengthening. FRP strengthening materials possess lower modulus' and linear stress-strain diagrams which significantly influence deflection and crack width patterns. Currently, there is limited information on serviceability issues of NSM strengthened structures compared to EBR strengthened structures.

ACl 318-99 (1999) recommended Branson's (Branson \& Metz, 1963) semi-empirical cubic equation for effective moment of inertia to compute immediate or short-term deflection. However, this equation is based on the behavior of RC beams reinforced with steel. Researchers have found that the effective moment of inertia for FRP reinforced beam is overestimated with this equation. $\mathrm{ACl}$ 440 (2006) modified this expression to make it applicable to FRP reinforced beams, although they explicitly state that further modification of the bond dependent coefficient in the equation is needed. Several authors (Benmokrane, 1996; Brown \& Bartholomew, 1996: Masmoudi, Theriault, \& Benmokrane, 1998. Pecce, Manfredi, \& Cosenza, 2000; Toutanji \& Saafi, 2000) have proposed modifications of this bond dependent coefficient, while others (Bischoff, 2005; Faza \& Ganga Rao, 1992; Smith \& Kim, 2011) have proposed a modified equivalent moment of inertia derived from curvatures. ACI 440 (2006) and the Canadian 
standard (CSA, 2002) have acknowledged these approaches. El-Mihilmy and Tedesco (2000) and Charkas, Rasheed, and Melhem (2003) have proposed a post yielding deflection model for FRP based EBR strengthened RC beams where the load-deflection curve is divided into three distinct linear stages, pre-cracking, cracking and post yielding. More recently, Visintin, Oehlers, Muhamad, and Wu (2013) have proposed a closed form solution based on the partial-interaction moment-rotation approach which can estimate all load levels from serviceability through to total collapse of a EBR strengthened RC beam.

The cracking behavior of FRP based EBR strengthened structures is quite unlike RC structures with steel due to the tension stiffening effects which develop at the steel-concrete and the FRP-concrete interface. As a result, crack widths and spacing become smaller compared to unstrengthened elements with the same service loading or same tension level in the steel (Ceroni \& Pecce, 2007, 2009). Currently the Italian guideline (CNR, 2006) recommends $0.5 \mathrm{~mm}$ as the allowable crack width for FRP reinforced members. This guideline also provides equations to measure characteristics of crack widths.

The assessment of deflection and crack width is required in order to meet the serviceability requirements. At the moment, a complete theoretical model for the deflection behavior of NSM strengthened RC structures is scarce. Finite Element Modeling (FEM) may provide a possible solution, although this method is quite expensive in terms of computational aspects, especially for geometrically complicated forms. The present crack width formulations do not allow for the prediction of crack width at each loading step in controlled laboratory conditions or even for actual field crack size. Therefore, there is a need for a simple and rapid, yet reliable and accurate alternative method to predict the serviceability of NSM strengthened structures. At present, various artificial intelligence techniques, such as Artificial Neural Networks (ANN) and Genetic Algorithms (GA), have been used in various FRP strengthened RC structures (Cevik, 2011; Nehdi, Chabib, \& Said, 2006). However, these techniques require extensive experimental results to optimize parameters, which is a challenging, labor intensive and time consuming process (Bashir \& Ashour, 2012; Kara, 2011). Conversely, the Fuzzy Logic Expert System (FLES) offers an effective solution as it depends on expert knowledge (Kim, Kim, \& Shin, 2014; Liu, Han, \& Lu, 2013; Nasir, Lim, Nahavandi, \& Creighton, 2014). It uses expert appraisals as well as a logical system closer to human reasoning rather than extensive experimental results. Cevik (2011) applied several soft computing techniques, such as neuro-fuzzy, genetic programming, stepwise regression and neural network to model the influence of FRP on confined concrete cylinders. The model was based on collected experimental data from open literature, which showed superior accuracy. Their formulation also conforms to the existing 10 models. Zheng, Li, and Wang (2011) predicted the delamination size and location of glass/epoxy laminate beams using a combination of fuzzy logic theory, neural networks and genetic algorithms. Modal frequencies were obtained from finite element analysis and the parameters were fed in this genetic fuzzy hybrid learning algorithm. The model demonstrated robust and promising applications in the structural health monitoring system. Nasrollahzadeh and Basiri (2014) developed a model to predict the shear strength of FRP reinforced RC structures using the Fuzzy Inference System (FIS). The study samples were 197 RC beams and slabs for which they utilized the subtractive clustering approach for partitioning the numerical data. The output of their model was only compared with the shear design guidelines (e.g., $\mathrm{ACI}$ and $\mathrm{CAN} / \mathrm{CSA}$ ). However, no work was found in the literature concerning flexural strengthened NSM-RC beams using FLES. Therefore, this study proposes an innovative approach based on FLES to predict the serviceability of RC structures flexurally strengthened with NSM reinforcement. This study consists of two approaches:

(1) The experimental method: Seven full sized RC beams were strengthened with CFRP and steel using the NSM technique. The beams were tested under four point bending load conditions. The deflection and crack width of steel and CFRP strengthened RC beams were measured with variable bonded lengths.

(2) The intelligent method: The study comprises deflection and crack width as the output parameters while applied force and bonded lengths are the input parameters for FLES. This system uses expert logic using IF-THEN rules, which connects the input and output variables with linguistic concepts.

The goal of the study was to determine the viability of using the FLES approach to achieve an accurate yet rapid prediction model for the deflection and crack width of NSM strengthened RC beams. It was expected that this approach would allow the predicted values to be obtained within a short period of time, which, in turn, would make it possible for a large number of alternative strengthening configurations to be evaluated, and thus beam specifications could be easily optimized for future use. The importance of the FLES approach is that it is possible to make flexural strengthened NSM-RC beam application, more viable and thus more attractive to potential users, such as design engineers and the Expert Systems with Applications (ESWA) community, etc.

\section{Experimental methodology}

\subsection{Test matrix}

Various parameters affect the flexural behavior of NSM strengthened beams. This study concentrates on the effect of length variation $(1600,1800$ and $1900 \mathrm{~mm}$ ) and the type of strengthening material (steel and CFRP bar) applied in strengthening the RC beams. A total of seven RC beam specimens were tested under static loading conditions in four point bending until failure. The specimens were divided into two main groups based on different strengthening material (CFRP and steel bar) and within the groups bond length was also varied. The test matrix is presented below in Table 1.

\subsection{Materials}

Ready mixed concrete was used for the construction of the RC beam specimens. Crushed stone, $20 \mathrm{~mm}$ in diameter, was used as coarse aggregate and natural river sand was used as fine aggregate. The concrete cube compressive strength at 28 days was $43.24 \mathrm{MPa}$ and cylinder strength was $35.63 \mathrm{MPa}$. Flexural strength was found to be $5.01 \mathrm{MPa}$. Compressive and flexural strengths of the concrete were determined according to BS EN (2009a), ASTM (2014) and BS EN (2009b). The dimensions of the cube, cylinder and prism were $100 \times 100 \times 100 \mathrm{~mm}, 200 \times 100 \mathrm{~mm}$ diameter and $500 \times 100 \times$ $100 \mathrm{~mm}$, respectively.

Deformed steel bars, $12 \mathrm{~mm}$ in diameter, were used for internal longitudinal reinforcement in the beams. The deformed bars were tested for tensile strength in the laboratory to confirm the mechanical properties supplied by the manufacturer. The yield stress and modulus of elasticity were found to be $400 \mathrm{MPa}$ and $200 \mathrm{GPa}$ respectively. $8 \mathrm{~mm}$ steel bars with yield stress of $380 \mathrm{MPa}$ and modulus of elasticity of $200 \mathrm{GPa}$ were used as shear reinforcement. $12 \mathrm{~mm}$ deformed steel bars with yield stress and modulus of elasticity of $520 \mathrm{MPa}$ and 200 GPa respectively, were used for NSM strengthening. 
Table 1

Test matrix.

\begin{tabular}{lllll}
\hline Sl. No. & Notation & Description & Groove size $(\mathrm{mm})$ & Total bonded length of strengthened bars $2 L(\mathrm{~mm})$ \\
\hline 1 & CB & Control RC beam (unstrengthened) & - \\
2 & N1.6F & NSM strengthened RC beam with 12 mm CFRP bar & $2 d_{b} \times 2 d_{b}$ & 1600 \\
3 & N1.6S & NSM strengthened RC beam with 12 mm steel bar & & 1600 \\
4 & N1.8F & NSM strengthened RC beam with 12 mm CFRP bar & & 1800 \\
5 & N1.8S & NSM strengthened RC beam with 12 mm steel bar & 1800 \\
6 & N1.9F & NSM strengthened RC beam with 12 mm CFRP bar & 1900 \\
7 & N1.9S & NSM strengthened RC beam with 12 mm steel bar & 1900 \\
\hline
\end{tabular}

$d_{b}=$ Diameter of NSM bar $(\mathrm{mm})$

Carbon-epoxy pultruded FRP (CFRP) bars $12 \mathrm{~mm}$ in diameter with a density of $1.65 \mathrm{~g} / \mathrm{mm}^{2}$ were also used for NSM strengthening in this study. These CFRP bars demonstrated linear elastic behavior up to failure. According to manufacturer's product result (LAMACO Inc.), the ultimate strength of the bars was found to be $2400 \mathrm{MPa}$ and the modulus of elasticity was $165 \mathrm{GPa}$. The surface of the CFRP bars were sand-coated to enhance bond performance.

An epoxy adhesive, Sikadur ${ }^{\circledR 3}$, was used to bond the NSM reinforcements to the concrete substrate for superior bond strength. The adhesive is composed of two components, a resin and a hardener, which were blended together in a ratio of 3:1 until an even gray color was attained. According to the manufacturer's results, the density was $1.65 \mathrm{~kg} /$ liter at $23^{\circ} \mathrm{C}$. The bond strength with steel was $21 \mathrm{MPa}$ according to DIN EN 24624 and with concrete was $4 \mathrm{MPa}$. The compressive, tensile and shear strength of this adhesive were $85-95 \mathrm{MPa}, 26-31 \mathrm{MPa}$ and $16-19 \mathrm{MPa}$ respectively at 7 days curing time and at $35^{\circ} \mathrm{C}$ temperature (Sikadur $-30,2014$ ).

\subsection{Procedure and beam preparation}

The beams were $2.3 \mathrm{~m}$ long with effective spans of $2 \mathrm{~m}$ and rectangular cross-sections with dimension of $125 \times 250 \mathrm{~mm}$. The beams were cast in the laboratory using ready mix concrete with the specifications given above. The tension reinforcements consisted of two $12 \mathrm{~mm}$ diameter ribbed bars with a ninety degree bend at both ends. Two $10 \mathrm{~mm}$ diameter ribbed bars were used in the compression zone as hanger bars and were run along the zones where the flexure moment is not constant. The shear reinforcements were made with $8 \mathrm{~mm}$ diameter ribbed steel bars spaced $90 \mathrm{~mm}$ apart. The beams were designed as under reinforced ( $\left.\rho=A_{s} / b_{d}=0.0085\right)$ beams to initiate failure in flexure in accordance with the $\mathrm{ACl}$ code. Fig. 1 displays the details of the beam configuration.

\subsection{Strengthening Procedure}

All the strengthened beam specimens had a single groove $(24 \times 24 \mathrm{~mm})$ cut along the beam length to accommodate a $12 \mathrm{~mm}$ diameter steel or CFRP bar. A special concrete saw with a diamond blade was used to create the grooves in the longitudinal direction on the tension side of the RC beam. A hammer and hand chisel were used to remove any remaining concrete lugs in the groove and to create a rough surface inside the groove. Debris and fine particles were also removed from the groove using an air brush. These steps were done to ensure appropriate bonding between the epoxy adhesive and the concrete. The groove was filled with epoxy adhesive to around $2 / 3$ of the groove depth.
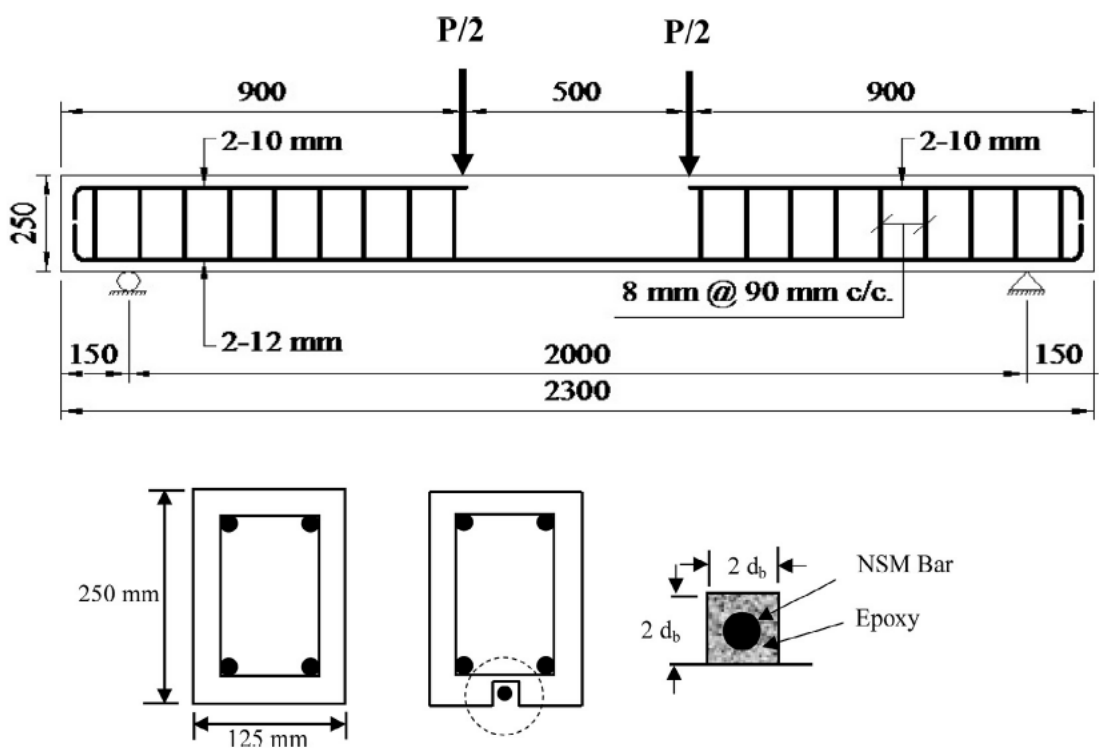

Fig. 1. Specimen design details. 


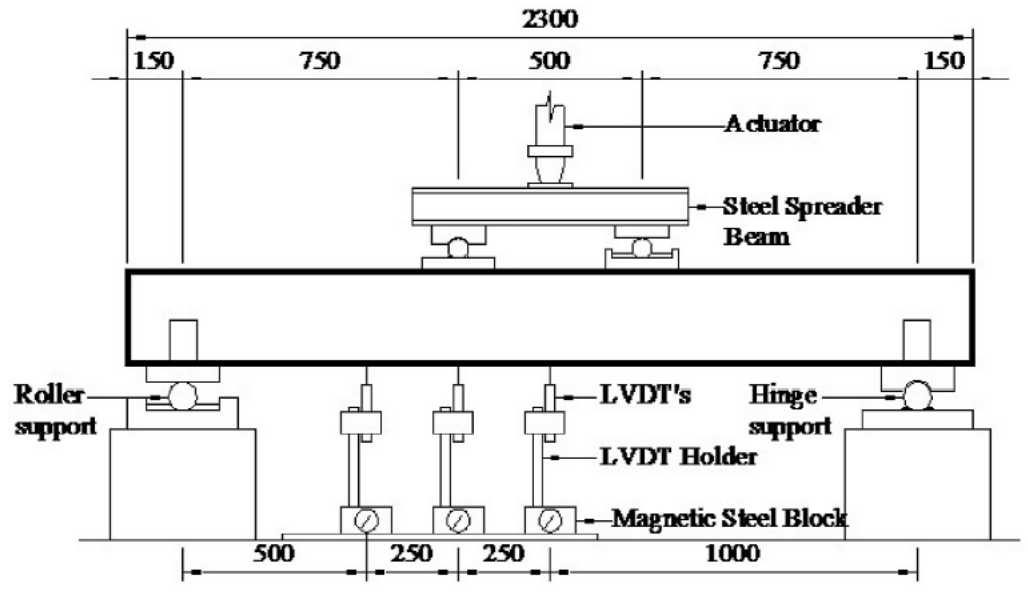

Fig. 2. Instrumentation and loading setup.

The CFRP or steel bar was gently inserted into the groove and pressed lightly to ensure the epoxy covered the bar. The remaining space in the groove was filled with epoxy and the surface levelled. This arrangement was left for one week in order to allow the epoxy to achieve full strength.

\subsection{Test setup and instrumentation}

A closed-loop Instron universal testing machine of $500 \mathrm{kN}$ capacity was used to apply four point loading on the prepared specimens. The machine was operated under load control mode with a loading rate of $5 \mathrm{kN} / \mathrm{min}$ up to yielding of the internal reinforcement of the RC beam specimens. After yielding, displacement control was applied up to failure with a rate of $1.8 \mathrm{~mm} / \mathrm{min}$.

The instrumentation of the beams is shown in Fig. 2. Three vertical Linear Variable Differential Transducers (LVDT) was affixed at the center, $250 \mathrm{~mm}$ from the center (under the spreader beam load point) and $500 \mathrm{~mm}$ from the center of the RC beam to measure the deflection at these different points. Strain gauges $5 \mathrm{~mm}$ in length were attached to the center of both the internal steel reinforcements and the NSM rods to measure tensile strains. Another $5 \mathrm{~mm}$ strain gauge was put $200 \mathrm{~mm}$ away from the cutoff point on the NSM bars. $30 \mathrm{~mm}$ strain gauges were placed on the top surface of the RC beam to measure concrete compressive strains. Demec disks were planted at the midspan of the beams along the depth to measure transverse strains. A Dino-Lite digital microscope was used to measure micro cracks on the surface of the beams.

\section{Prediction model design}

3.1. Serviceability recommendations from existing codes and guidelines

In early RC designing serviceability issues were dealt with indirectly. However, serviceability is now considered a major issue in designing a structure. Among the two limit states (strength and serviceability), the serviceability limit state refers to the performance of structures under normal service loads and is concerned with the uses and/or occupancy of structures. Serviceability is measured by considering the magnitudes of deflections, cracks, and vibrations of structures. In general, ACI 318-05 provisions for deflection control are concerned with deflections that occur at service levels under immediate and sustained static loads. The present study only concentrates on short term or immediate deflection.

\subsubsection{Deflection Measurement}

The empirically calibrated Branson's equation represents the transition from uncracked gross moment of inertia $\left(I_{g}\right)$ to the transformed moment of inertia $\left(I_{\sigma}\right)$. ACl 318-99 (1999) adopted this Eq. (1) for effective moment of inertia $\left(I_{e}\right)$ to estimate the immediate deflection of steel reinforced RC beams.

$I_{e}=I_{c r}+\left(I_{g}-I_{c r}\right)\left(\frac{M_{c r}}{M_{a}}\right)^{3}$

Here, $M_{a}$ is the service moment and $M_{c r}$ is the cracking moment. However, researchers have found that this Eq. (1) overestimates the $I_{e}$ of a FRP reinforced beam due to the linear elastic behavior of FRP material. Gao, Benmokrane, and Masmoudi (1998) proposed a modified version of Branson's equation (2) for FRP reinforced beams.

$I_{e}=I_{c r}+\left(\beta_{d} I_{g}-I_{c r}\right)\left(\frac{M_{c r}}{M_{a}}\right)^{3}$

$\beta_{d}=\alpha_{b}\left[\frac{E_{f}}{E_{s}}+1\right]$

Here, $\alpha_{b}$ is a bond dependent coefficient and $\mathrm{ACl}$ recommends its value be 0.5 for all FRP bar types until a more precise value is determined from further research.

The Italian guideline (CNR, 2006) has suggested that the curvature diagram of the FRP reinforced member be integrated following the proposed Eurocode 2 model. Non-linear analysis can be used to consider this diagram by considering both the tension stiffening and cracking of the concrete. The deflection $f$ can be calculated using the following Eq. (3).

$f=f_{1} \beta_{1} \beta_{2}\left(\frac{M_{c r}}{M_{\max }}\right)^{m}+f_{2}\left[1-\beta_{1} \beta_{2}\left(\frac{M_{c r}}{M_{\max }}\right)^{m}\right]$

where, $f_{1}=$ deflection of the uncracked section; $f_{2}=$ deflection of the transformed cracked section; $\beta_{1}=0.5$, which is the value of FRP bar bond properties; $\beta_{2}=$ coefficient used for the duration of loading ( 1 for short term and 0.5 for long term or cyclic loading); 
$M_{\max }=$ maximum moment; $M_{c r}=$ cracking moment; and $m=\mathrm{a}$ coefficient equal to 2 .

To avoid inelastic deformations of RC members with nonprestressed external FRP reinforcement, the existing internal steel reinforcement should be prevented from yielding under service load levels, especially for members subjected to cyclic loads. For this reason, $\mathrm{ACl}$ has set two Eqs. (4) and (5) for the stress in steel and concrete under service loading ( $\mathrm{ACI} 440.2 \mathrm{R}-08,2008$ ). They are as follows.

$f_{s, s} \leqslant 0.80 f_{y}$

$f_{c s} \leqslant 0.45 f^{\prime}$

The stress level in the steel reinforcement can be calculated based on a cracked-section analysis of the FRP-strengthened reinforced concrete section (Fig. 3), as indicated in the following Eq. (6):

$f_{s, s}=\frac{\left[M_{s}+\varepsilon_{b i} A_{f} E_{f}\left(d_{f}-\frac{k d}{3}\right)\right](d-k d) E_{s}}{A_{s} E_{s}\left(d-\frac{k d}{3}\right)(d-k d)+A_{f} E_{f}\left(d_{f}-\frac{k d}{3}\right)\left(d_{f}-k d\right)}$

Here, $M_{s}$ is equal to the moment due to all sustained loads (dead loads and the sustained portion of the live load) plus the maximum moment induced in a fatigue loading cycle. Under service loading conditions, within the elastic response range of the member, the FRP stress level can be computed using the following Eq. (7).

$f_{f, s}=f_{s, s}\left(\frac{E_{f}}{E_{s}}\right) \frac{d_{f}-k d}{d-k d}-\varepsilon_{b i} E_{f}$

\subsubsection{Crack width}

According to $\mathrm{ACl}$, allowable crack width in FRP reinforced structures is wider than in steel reinforced concrete beams as FRP is a corrosion resistant material. The well-known Gergely and Lutz (1973) crack width equation is mainly used for steel reinforced structures and requires modification when applied to FRP reinforced members (Gao et al., 1998). The original Gergely-Lutz equation (8) with SI units is as follows.

\section{$w=0.0113 \beta_{h} f_{s} \sqrt[3]{d_{c} A}$}

Here, the crack width $w$ is expressed in $\mathrm{mm}$. Wang and Salmon (1992) pointed out the fact that instead of stress in the tensile reinforcement, strain is the guiding factor that varies proportionately with crack width. Based on this concept, the Gergely-Lutz equation has been modified by replacing the steel strain $\varepsilon_{s}$ with the FRP strain, $\varepsilon_{f}=\frac{f_{f}}{E_{\text {. }}}$. A bond quality coefficient $k_{b}$ is also introduced to Eqs. (9) and (10), which becomes as follows.
$w=\frac{2.2}{E_{f}} \beta_{h} k_{b} f_{f} \sqrt[3]{d_{c} A}$

$f_{f}=M_{s} \frac{n_{f} d(1-k)}{I_{c r}}$

Here, $k_{b}<1$ (when FRP bond behavior is superior to steel); $k_{b}=1$ (when FRP bond behavior is similar to steel); $k_{b}>1$ (when FRP bond behavior is inferior to steel); $E_{f}=$ modulus of elasticity of FRP $(\mathrm{MPa}) ; \beta_{h}=$ ratio of the distance between the neutral axis and the tension face to the distance between the neutral axis and the centroid of reinforcement; $f_{f}=$ stress level in FRP $(\mathrm{MPa}) ; d_{c}=$ thickness of cover from the tension face to the center of the closest ba $(\mathrm{mm}) ; A=$ the effective tension area of concrete around the main reinforcement divided by the number of bars $\left(\mathrm{mm}^{2}\right)$. The $\mathrm{ACI}$ committee recommends a value of 1.2 for deformed FRP bars, if $\mathrm{K}_{\mathrm{b}}$ is unknown. Eurocode 2 (EC2-04, 2004) has proposed an expression for calculating the characteristic value of crack width. Eq. (11) is as follows.

$W_{k}=S_{r, \max }\left(\varepsilon_{s m}-\varepsilon_{c m}\right)$

Maximum crack spacing can be calculated from the following Eq. (12),

$S_{r, \max }=3.4 c+0.425 k_{1} k_{2} \frac{\phi}{\mu_{s}}$

The difference between the mean steel and the concrete strain between cracks can be calculated using Eq. (13),

$\varepsilon_{s m}-\varepsilon_{c m}=\frac{\sigma_{s}}{E_{s}}-k_{t}\left[\frac{f_{c t m} A_{c, e f f}}{E_{s} A_{s}}+\frac{f_{c t m}}{E_{c m}}\right]$

where, $k_{t}=$ factor of load duration; $A_{\underline{c}, \text { eff }}=$ effective area of concrete in tension; $k_{1}$ = bond coefficient; $k_{2}=$ type of loading; = diameter of bar; $\mu_{s}=$ ratio of the internal steel reinforcement to the effective area of concrete in tension. In the updated Eurocode 2, the bond relationship is present only in the crack spacing formulation, in a simplified way by the coefficient $k_{1}$, while in the tension stiffening term $\left(\varepsilon_{s m}-\varepsilon_{c m}\right)$ the concrete tensile strength appears and the surface type of bars is neglected (Ceroni \& Pecce, 2009).

\subsection{Fuzzy inference system}

The basic configuration of a fuzzy logic expert system (FLES) comprises of four principal components (Passino, Yurkovich, \& Reinfrank, 1998). They are:

(1) Fuzzification - which takes crisp numeric inputs and converts them into the fuzzy form needed by the decisionmaking logic. The first task in fuzzification interfaces is the

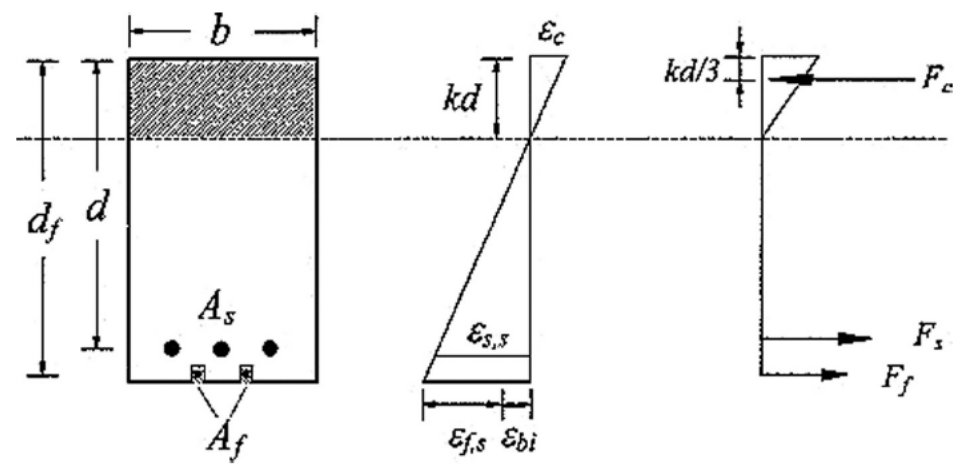

Fig. 3. Elastic strain and stress distribution. 
selection of input and output variables. After that all input and output numeric variables have to be defined in linguistic terms such as low, medium, high and so on. Subsequently, membership functions for all input and output variables have to be formed. The central concept of fuzzy set theory is membership functions, which represent numerically to what degree an element belongs to a set. A membership function is typical a curve that converts the crisp numerical value of input variable into the fuzzy number within a range from 0 to 1 , representing the belongingness of the input to a fuzzy set. There are different forms of membership functions such as triangle, trapezoid, and Gaussian functions. The selection of membership functions and their formations is based on system knowledge, expert's appraisals, and experimental conditions.

(2) Rule base - which holds a set of if-then rules that quantify the knowledge that human experts have amassed about solving a specific problem. Moreover, fuzzy rules are the heart of the fuzzy expert system which determines the relationship between input and output of the model. This performs as a source to the decision making logic. Moreover, it consists of a data base and a rule base. In the fuzzy knowledge base system, knowledge is represented by if-then rules. Fuzzy rules consist of two parts: an antecedent part stating conditions on the input variables and a consequent part describing the corresponding values of output variables. For instance, in the case of three inputs $\mathrm{P}, \mathrm{Q}$, and $\mathrm{R}$, and one output $Z$, which have the linguistic variables of very low, medium, and low medium for $\mathrm{P}, \mathrm{Q}$ and $\mathrm{R}$ respectively and medium for $\mathrm{Z}$, then development of fuzzy inference rules can be demonstrated as follows:

If $P$ is very low and $Q$ is medium, and $R$ is low medium then $Z$ is medium.

(3) Inference - which creates the control actions according to the information provided by the fuzzification module and by applying knowledge. It plays a central role in a fuzzy logic model due to its ability to create human decision making and deduce fuzzy control actions as per the information provided by the fuzzification module by applying knowledge about how to control best the process. Three types of fuzzy inference systems (FIS) have been widely employed in various applications: Mamdani, Sugeno and Tsukamoto fuzzy models (Cevik, 2011). The differences between these three fuzzy inference systems are due to the consequences of their fuzzy rules, and thus their aggregation and defuzzification procedures differ accordingly (Jang, Sun, \& Mizutani, 1997). The Mamdani-type FIS uses the technique of defuzzification of a fuzzy output and has output membership functions (Mamdani \& Assilian, 1975). This FIS is widely accepted for capturing expert knowledge (Kaur \& Kaur, 2012). The Mamdani-type FIS describes the expertise in more intuitive, and more human like manner. Most commonly, the Mamdani max-min fuzzy inference mechanism is used because it ensures a linear interpolation of the output between the rules.

For instance, in case of tree-inputs and single-output fuzzy inference system, it can be shown (Fig. 4) as below.

where applied load $(F)$ and rod length $(L)$ are in input side and deflection for steel bar strengthened beams (DS), crack width for steel bar strengthened beams (WS), deflection for CFRP bar strengthened beams (DF) and crack width for CFRP bar strengthened beams (WF) are on output side.

(4) Defuzzification - which calculates the actual output, i.e. converts fuzzy output into a precise numerical value (crisp value). The conversion of a fuzzy set to a single crisp output on which action can be taken is called defuzzification. The defuzzification interface combines the conclusions reached by the decision-making logic and converts the fuzzy output into a precise crisp numeric value. There are several methods of defuzzification, such as centroid, center of sum, mean of maxima and left-right maxima. Most commonly, the center of gravity (centroid) defuzzification method is used, since this operator assures a linear interpolation of the output between the rules.

\subsection{Implementation of fuzzy logic}

In the FLES model created for this study there are two input parameters, applied load $(F)$ and rod length $(L)$. From the laboratory investigation it has been experienced that these two parameters could significantly influence four output parameters: deflection for steel bar strengthened beams (DS), crack width for steel bar strengthened beams (WS), deflection for CFRP bar strengthened beams (DF) and crack width for CFRP bar strengthened beams (WF) (Table 2). Actually, the input parameters of $F$ and $L$ might be less or more in simulating the intensity and magnitude of load and rod length. The load varied within the range from 0 to $100 \mathrm{kN}$ and the rod length varied between 1600 and $1800 \mathrm{~mm}$. In this instance, eleven linguistic variables for Load $(F)$ and four linguistic variables for Length $(L)$ were considered. For fuzzification,

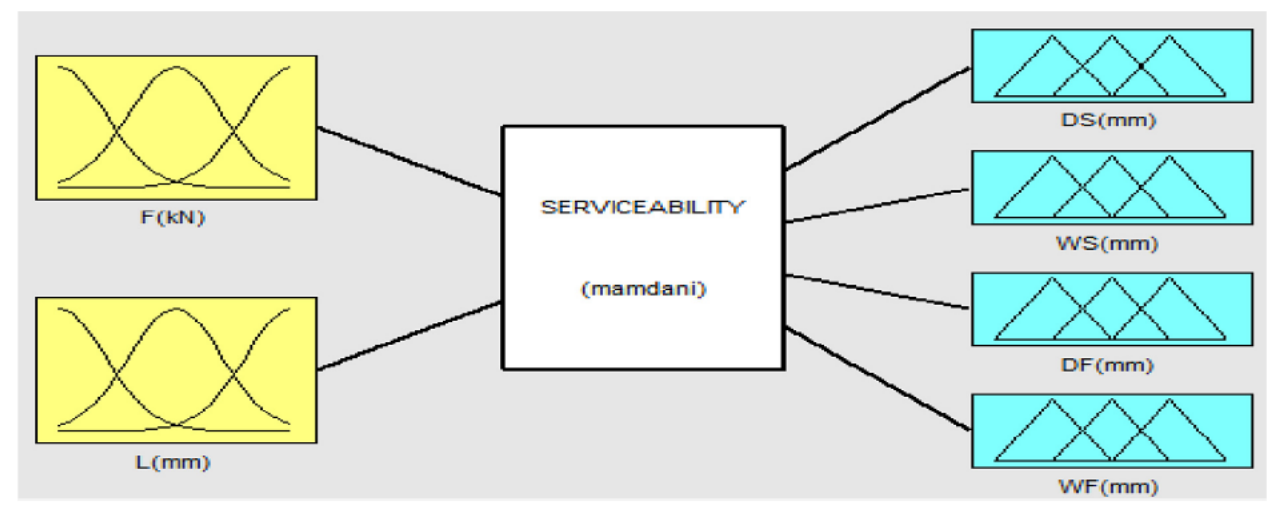

Fig. 4. Fuzzy inference mechanisms. 
Table 2

Fuzzy inference rules.

\begin{tabular}{|c|c|c|c|c|c|c|}
\hline \multirow[t]{2}{*}{ Rule No. } & \multicolumn{2}{|c|}{ Input variables } & \multicolumn{4}{|c|}{ Output variables } \\
\hline & $F$ & $L$ & DS & ws & $D F$ & $W F$ \\
\hline 1 & VVL & VL & L1 & L1 & L1 & L1 \\
\hline 7 & VL & M & L2 & L1 & L2 & L1 \\
\hline 12 & L & $\mathrm{H}$ & L2 & L2 & L3 & L3 \\
\hline 18 & M & L & $\mathrm{L} 4$ & L7 & L5 & L8 \\
\hline 25 & VHM & $\mathrm{VL}$ & L6 & L9 & L9 & L12 \\
\hline 36 & $\mathrm{H}$ & $\mathrm{H}$ & L7 & L8 & L9 & L10 \\
\hline 44 & VVH & $\mathrm{H}$ & L9 & LS & L10 & L11 \\
\hline
\end{tabular}

the input variable $\mathrm{F}$ was given eleven possible linguistic variables, namely very very low (VVL), very low (VL), low (L), High low (HL), low medium (LM), medium (M), high medium (HM), medium high $(\mathrm{MH})$, high $(\mathrm{H})$, very high $(\mathrm{VH})$, and very very high (VVH), and for input variable L four linguistic variables were used, very low $(\mathrm{VL})$, low $(\mathrm{L})$, medium $(\mathrm{M})$, and high $(\mathrm{H})$. The linguistic variables used for the output variables were levels 1 to 12 for $D S$ and $D F$, and levels 1 to 16 for WS and WF, respectively. If more input variables were considered the fuzzy inference rules would become more complex and vice versa. Therefore, the number of input variables were reasonable to achieve suitable output from the proposed model. A Mamdani max-min inference approach and the center of gravity defuzzification method were applied as these operators assure a linear interpolation of the output between the rules (Hossain, Rahman, \& Mohiuddin, 2012). Fig. 4 displays the fuzzy inference system in the case of two inputs and four outputs. The units of the input and output variables are $\mathrm{kN}$ for $F$ and $\mathrm{mm}$ for $L, D S, W S, D F$ and WF. A total of 44 fuzzy inference rules were formed based on expert knowledge and past experience. Some of the rules are shown in Table 2. An example is illustrated here concerning how the values of the last four columns of fuzzy inference rules (Table 2) are determined.

Rule 1: If applied force $(F)$ is very very low (VVL), and rod length $(L)$ is very low (VL) then deflection for steel bar strengthened beams (DS) is level 1 (L1), crack width for steel bar strengthened beams (WS) is level 1 (L1), deflection for CFRP bar strengthened beams (DF) is level 1 (L1), and crack width for CFRP bar strengthened beams (WF) is level 1 (L1)

Rule 36: If applied force $(F)$ is high $(\mathrm{H})$, and rod length $(L)$ is high (H) then deflection for steel bar strengthened beams (DS) is level 7 (L7), crack width for steel bar strengthened beams (WS) is level 8 (L8), deflection for CFRP bar strengthened beams (DF) is level 9
(L9), and crack width for CFRP bar strengthened beams (WF) is level 10 (L10).

There is a level of membership for each linguistic word that applies to an input variable. Fuzzifications of the input variables were made by using the following Eqs. (14)-(19):

$$
\begin{aligned}
& F\left(i_{1}\right)=\left\{\begin{array}{ll}
i_{1} ; & 0 \leq i_{1} \leq 100 \\
0 ; & \text { otherwise }
\end{array}\right\} \\
& L\left(i_{2}\right)=\left\{\begin{array}{ll}
i_{2} ; & 1600 \leqslant i_{2} \leqslant 1900 \\
0 ; & \text { otherwise }
\end{array}\right\} \\
& D S\left(o_{1}\right)=\left\{\begin{array}{ll}
0_{1} ; & 0 \leqslant 0_{1} \leqslant 16 \\
0 ; & \text { otherwise }
\end{array}\right\} \\
& W S\left(o_{2}\right)=\left\{\begin{array}{ll}
0_{2} ; & 0 \leqslant 0_{2} \leqslant 0.64 \\
0 ; & \text { otherwise }
\end{array}\right\} \\
& D F\left(o_{3}\right)=\left\{\begin{array}{ll}
0_{3} ; & 0 \leqslant 0_{3} \leqslant 16 \\
0 ; & \text { otherwise }
\end{array}\right\} \\
& W F\left(o_{4}\right)=\left\{\begin{array}{ll}
0_{4} ; & 0 \leqslant 0_{4} \leqslant 0.64 \\
0 ; & \text { otherwise }
\end{array}\right\}
\end{aligned}
$$

where, is the first input variable load $(F)$, is the second input variable length $(L)$ and $o_{1}, o_{2}, o_{3}$ and $o_{4}$ are the output variables $D S, W S, D F$ and $W F$ respectively. Prototype triangular fuzzy sets for the fuzzy variables were set up using MATLAB Fuzzy Toolbox. Among the many membership functions, triangular, trapezoidal, piecewise linear and Gaussian are those mostly used. These membership functions are chosen based on the researcher's past experience and ease of application. Moreover, the triangular membership functions provide a faster and easier solution, as, in the symmetric condition, the center of gravity is at the apex of the triangle, which makes computational calculation easier (Mendel, 1995). The membership values obtained from the above formulas are shown in Fig. 5 for two input variables.

To demonstrate the fuzzification process, linguistic expressions for the triangular membership functions can be described using following Eq. (20):

$$
\mu_{\text {triangle }}\left(x, c_{1}, c_{2}, c_{3}\right)=\left\{\begin{array}{ll}
0 ; & x \leqslant c_{1} \\
\frac{x-c_{1}}{c_{2}-c_{1}} ; & c_{1} \leqslant x \leqslant c_{2} \\
\frac{c_{3}-x}{c_{3}-c_{2}} ; & c_{2} \leqslant i_{1} \leqslant c_{3} \\
0 ; & x \geqslant c_{3}
\end{array}\right\}
$$

where, $x$ is the input and output variable; $c_{1}, c_{2}$ and $c_{3}$ are the coefficients of membership functions.

Linguistic expressions and membership functions of load $(F)$ and length $(L)$ obtained from the developed rules and above formula [Eqs. (14) and (15)] for HM and $\mathrm{H}$ are presented as follows.

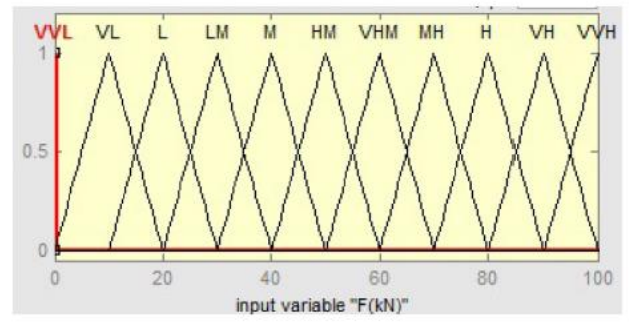

(a) Input variable " $\mathrm{F}(\mathrm{kN})$ "

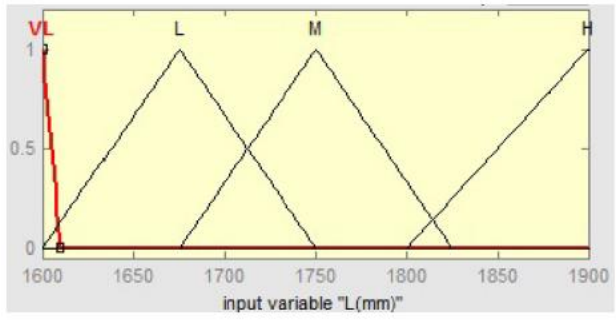

(b) Input variable "L (mm)"

Fig. 5. Membership functions of input variables (a) load $-F(\mathrm{kN})$ and (b) NSM length $-L(\mathrm{~mm})$.

Link to Full-Text Articles :

http://www.sciencedirect.com/science/article/pii/S0957417414004746 